\title{
SOX2 is essential for in vivo reprogramming of seminoma-like TCam-2 cells to an embryonal carcinoma-like fate
}

\author{
Daniel Nettersheim ${ }^{1}$, Alena Heimsoeth ${ }^{1}$, Sina Jostes ${ }^{1}$, Simon Schneider ${ }^{1}$, Martin \\ Fellermeyer ${ }^{1}$, Andrea Hofmann ${ }^{2}$, Hubert Schorle ${ }^{1}$ \\ ${ }^{1}$ Institute of Pathology, Department of Developmental Pathology, University of Bonn Medical School, Bonn, Germany \\ ${ }^{2}$ Institute of Human Genetics, University of Bonn Medical School, Bonn, Germany \\ Correspondence to: Hubert Schorle, email: Hubert.Schorle@ukb.uni-bonn.de
}

Keywords: TCam-2, seminoma, embryonal carcinoma, reprogramming, SOX2/SOX17

Received: March 22, 2016

Accepted: May 19, 2016

Published: June 07, 2016

\section{ABSTRACT}

Type II germ cell cancers (GCC) are divided into seminomas, which are highly similar to primordial germ cells and embryonal carcinomas (EC), often described as malignant counterparts to embryonic stem cells.

Previously, we demonstrated that the development of GCCs is a highly plastic process and strongly influenced by the microenvironment. While orthotopic transplantation into the testis promotes seminomatous growth of the seminoma-like cell line TCam-2, ectopic xenotransplantation into the flank initiates reprogramming into an EC-like fate.

During this reprogramming, BMP signaling is inhibited, leading to induction of NODAL signaling, upregulation of pluripotency factors and downregulation of seminoma markers, like SOX17. The pluripotency factor and EC-marker SOX2 is strongly induced.

Here, we adressed the molecular role of SOX2 in this reprogramming. Using CRISPR/Cas9-mediated genome-editing, we established SOX2-deficient TCam-2 cells. Xenografting of SOX2-deficient cells into the flank of nude mice resulted in maintenance of a seminoma-like fate, indicated by the histology and expression of OCT3/4, SOX17, TFAP2C, PRDM1 and PRAME. In SOX2-deficient cells, BMP signaling is inhibited, but NODAL signaling is not activated. Thus, SOX2 appears to be downstream of BMP signaling but upstream of NODAL activation. So, SOX2 is an essential factor in acquiring an EC-like cell fate from seminomas.

A small population of differentiated cells was identified resembling a mixed nonseminoma. Analyses of these cells revealed downregulation of the pluripotency and seminoma markers OCT3/4, SOX17, PRDM1 and TFAP2C. In contrast, the pioneer factor FOXA2 and its target genes were upregulated, suggesting that FOXA2 might play an important role in induction of non-seminomatous differentiation.

\section{INTRODUCTION}

All invasive type II germ cell cancers (GCC) arise from a common precursor lesion called germ cell neoplasia in situ (GCNIS) [1, 2]. However, the GCC subtypes seminoma and embryonal carcinoma (EC) show important differences regarding gene expression, growth and differentiation abilities. While seminomas grow as an undifferentiated cell mass, ECs exhibit features of totipotency and are capable of differentiation into all three germ layers (teratomas) and extra-embryonal tissues (yolk-sac tumor, choriocarcinoma). ECs and seminomas express the pluripotency markers NANOG and OCT3/4, but SOX2 is detected in ECs only and is thought to be compensated by SOX17 in seminomas [3]. In fact, SOX2 and SOX17 serve as markers for diagnostic discrimination between seminomas and ECs.

In a previous study, we demonstrated that xenografting of seminoma-like TCam-2 cells leads to inhibition of BMP signaling, which initiates reprogramming of TCam-2 into an EC-like fate $[1,4,5]$. This reprogramming process was accompanied by strong 
upregulation of 6 genes classified as initial drivers of the reprogramming process, i. e. GDF3, DPPA3, NODAL, $D N M T 3 B, G A L$ and $A K 3 L 1$. The changes in gene expression inversely correlated to the DNA methylation (5mC) levels within corresponding genomic loci [6]. Additionally, many pluripotency and EC associated genes, like SOX2, ZIC3, ZFP42, LIN28, SALL4 and PRDM14 were upregulated without correlating to changes in their 5mC status [7], while seminoma markers $S O X 17, c K I T$, PRDMI and PRAME were downregulated. Once TCam-2 adapted to an EC-like fate, BMP signaling recovered to a level lower than in parental TCam-2 and the newly acquired state was (epi)genetically stabilized by an autoregulatory NODAL signaling loop and strongly increased $5 \mathrm{mC}$ levels, silencing expression of seminoma-associated genes.

As part of the four Yamanaka factors, the pluripotency factor SOX2 is an essential transcription factor for reprogramming of various cells, like fibroblasts to an induced pluripotent state. In murine embryonic stem cells (mESC), Sox 2 complexes with Oct $3 / 4$ and binds to a canonical motif, thereby driving the expression of pluripotency genes [8]. Overexpression of Sox 17 is able to replace Sox 2 in the complex with Oct3/4, leading to a change in target site selection to a compressed binding motif [1]. We speculated that during reprogramming of TCam-2 the strong increase in SOX2 protein levels and downregulation of SOX17 leads to a switch to promoters harboring the canonical motif found in pluripotency genes. Furthermore, we postulated that during the seminoma to EC transition, inhibition of BMP signaling leads to derepression of $S O X 2$, restoring the classical pluripotency circuitry found in ECs and ESCs, subsequently leading to upregulation of $Z I C 3$, which in turn helps to maintain NODAL signaling $[7,9]$.

In this study, we analyzed the role of the pluripotency factor SOX2 in the in vivo reprogramming of TCam-2 to an EC-like cell fate. Therefore, we generated SOX2 knock out TCam-2 cells by utilizing the CRISPR/ Cas9 system and xenografted these cells into the flank of nude mice. After six weeks of in vivo growth, tumors were isolated and analyzed. Interestingly, TCam-2 cells did not acquire features of an EC, implicating that SOX2 is essential for the transition of TCam-2 cells to an EC-like cell state. Neither upregulation of EC-related pluripotency and epigenetic reprogramming factors, nor induction of NODAL or WNT signaling was detected. Additionally, global $5 \mathrm{mC}$ levels remained unaffected and expression of seminoma-associated genes SOX17, PRAME, TFAP2C, $P R D M 1$ and $C K I T$ was maintained. Nevertheless, a small subpopulation initiated differentiation into a mixed nonseminoma, demonstrating that in vivo the seminoma-like cell fate cannot be maintained for longer than 6 weeks. This differentiation was accompanied by upregulation of the pioneer factor FOXA2, which interacts with AFP, ALB, CDX1, DKK1, DLK1, PITX2, TTR, EOMES, apolipoproteins and fibrinogens. So, we hypothesize that this non-seminomatous in vivo differentiation of TCam-2 is triggered by FOXA2.

\section{RESULTS}

In a previous study, we demonstrated that TCam-2 cells are able to transit into an EC-like state when being xenografted into the flank of nude mice [10]. Analyses revealed that the somatic microenvironment inhibits BMP signaling, which is the initial step in the reprogramming process of TCam-2 cells, leading to induction of NODAL signaling and expression of EC-related genes as well as globally increased $5 \mathrm{mC}$ levels (in vitro: $18 \%$; 6 weeks in vivo: $70 \%$ ) [10]. During this reprogramming, we found a very rapid (after 1 week of in vivo growth) and strong upregulation of the transcription factor SOX2 $\left(\log _{2} 2.16\right.$ fold) and in parallel downregulation of SOX17 ( $\log _{2} 3.75$ fold) [10]. Due to the importance of SOX2 in cellular reprogramming and maintenance of pluripotency, we were interested in the role of SOX2 in the reprogramming of TCam-2. Therefore, we generated SOX2 knock out TCam-2 clones (TCam-2- $\triangle \mathrm{SOX} 2$ ) by utilizing the CRISPR/Cas9 technique. We simultaneously transfected TCam-2 cells with pX330 vector encoding for three different guide RNAs (gRNA) directed towards the SOX2 coding region (Figure $\mathrm{S} 1 \mathrm{~A}$ ). In parallel, a GFP-coding plasmid was transfected to identify clones that presumably have taken up the gRNA-pX330 plasmids. Two days after transfection, single GFP expressing cells were picked and clonally expanded. A PCR analysis revealed that all TCam-2- $\triangle$ SOX2 subclones (1 - 5) harbour SOX2 deletions on both alleles and show no band corresponding to the wildtype SOX2 sequence (Figure S1A, S1B). Expression of pluripotency and seminoma key factors was not significantly different between parental TCam-2 and TCam-2- $\Delta$ SOX2 clones, suggesting that a CRISPR/Cas9mediated depletion of SOX2 does not lead to off-target effects, impinging on the undifferentiated and seminomalike nature of TCam-2 cells (Figure S1C, S1D).

First, we xenografted a TCam-2- $\triangle \mathrm{SOX} 2$ clone into the flank of nude mice and analyzed the tumor tissue after one week of in vivo growth. Macroscopically, the tumor tissues presented as a uniform mass and microscopically showed typical seminoma-morphology i. e. big roundish cells with big nuclei and a clear cytoplasm (Figure S2A, S2B). Additionally, the tumor cells were positive for OCT3/4 and TFAP2C and negative for SOX2 (Figure S2B). By an expression microarray, we analyzed the global gene expression profile of TCam-2- $\triangle \mathrm{SOX} 2$ cells 1 week after xenografting and TCam-2 in vitro. On a global scale, the expression profile of TCam-2- $\triangle \mathrm{SOX} 2$ cells one week after xenografting and TCam-2 in vitro was highly similar (Figure S2C). In more detail, the expression intensities of typical seminoma- and EC-markers (all expressed at very 
low levels) were highly comparable between xenografted TCam-2- $\triangle \mathrm{SOX} 2$ cells and TCam- 2 in vitro suggesting that 1 week after xenografting the TCam-2- $\triangle \mathrm{SOX} 2$ clones do not differ considerably from in vitro cultivated TCam-2 with regard to gene exression (Figure S2D). We found 296 differentially expressed genes (247 upregulated, 49 downregualted). Among the upregulated genes, we found no EC marker and among the downregulated genes, no PGC/GCNIS/seminoma marker was found (Data S1A). Interestingly, among the downregulated genes IDI (fold change $\log _{2} 1.84$ ) and $I D 3$ were found, suggesting that similarly to transiting TCam-2, BMP signaling is also inhibited in TCam-2- $\triangle \mathrm{SOX} 2$ early after xenografting (Data S1A) [10].

Next, we asked if the TCam-2- $\triangle \mathrm{SOX} 2$ clones maintain their seminomatous nature for longer than 1 week in vivo. Therefore, we xenografted the 5 TCam-2- $\triangle \mathrm{SOX} 2$ clones into the flank of nude mice and monitored tumor growth for six weeks. As controls, parental TCam-2 and 2102EP were transplanted. 2102EP cells express typical EC- and pluripotency factors, but are nullipotent and thus less prone to differentiation than other EC cell lines. So, 2102EP cells closely resemble an undifferentiated EC in vitro and in vivo. After six weeks, tumors were recovered and analyzed. Macroscopically, the 2102EP grew into the largest tumor, followed by the TCam-2 tumor, which should have adapted an EC-like cell fate at this time point (Figure 1A) [10]. One TCam-2- $\Delta$ SOX2 tumor (1) showed a similar size as the TCam-2 tumor, while TCam-2- $\triangle \mathrm{SOX} 2$ tumors 2 - 5 were considerably smaller (Figure 1A). In contrast to the 2102EP and TCam-2 tumor, the TCam-2- $\triangle \mathrm{SOX} 2$ tumors appeared as a homogeneous mass (Figure 1A). HE staining of TCam-2- $\Delta$ SOX2 tumors revealed a typical seminomalike morphology (Figure 1B). In contrast, tumors from xenografted 2102EP and TCam-2 cells displayed a typical EC morphology, i. e. small polygonal and eosinophilic cells with hard to distinguishable cellular borders (Figure 1B).

To analyze genome-wide deregulation in gene expression, we performed microarray analyses of the TCam-2- $\triangle$ SOX2 tumors. Tumors from xenografted TCam2 and 2102EP cells as well as in vitro cultivated TCam-2 served as control. We compared the global expression profiles using unsupervised hierarchical clustering and illustrated the data as a heatmap (samples labeled in green) (Figure 1C). Additionally, we meta-analyzed microarray expression data from a previous study elucidating the reprogramming of TCam-2 to an EC-like fate in vivo (all samples labeled in black) [10]. The hierarchical clustering and heatmap analysis demonstrated that all five TCam-2- $\triangle \mathrm{SOX} 2$ clones clustered closely to each other, demonstrating good reproducibility of the analysis. Further, the tumor from xenografted 2102EP clustered to the 2120EP in vitro and in vivo samples from our previous study and the tumor samples from xenografted TCam-2 clustered to the tumor tissues from TCam-2 after 4 and 6 weeks of in vivo growth. Finally, our TCam-2 in vitro control clustered to our TCam-2 control from our previous study and to TCam-2 cells grown in vivo for 1 and 2 weeks, which are still closely related to TCam- 2 in vitro. Interestingly, the TCam-2- $\Delta \mathrm{SOX} 2$ clones clustered more closely to the TCam- 2 control cells $(1 \mathrm{w}, 2 \mathrm{w}$, in vitro $)$ than to the 2102EP ( $4 \mathrm{w}, 8 \mathrm{w}$, in vitro) or reprogrammed TCam-2 samples ( $4 \mathrm{w}, 6 \mathrm{w}$ ). Thus, the TCam-2- $\triangle \mathrm{SOX} 2$ clones display a global gene expression profile more similar to a seminoma than an EC. Nevertheless, the TCam-2- $\triangle \mathrm{SOX} 2$ clones deregulated a cluster of genes, which is not altered in all other analyzed samples (Figure 1C, yellow box), indicating that the TCam-2$\triangle \mathrm{SOX} 2$ clones also underwent changes in gene expression.

Next, we screened the microarray data for expression of key factors driving the seminoma to EC transition, pluripotency factors, seminoma markers, signaling pathway-related genes and epigenetic factors (Figure 2A, Data S1B) [10]. qRT-PCR analyses verified all detected deregulation in gene expression (Figure 2B). In contrast to xenografted TCam-2 and 2102EP cells, the TCam-2- $\triangle \mathrm{SOX} 2$ clones showed no upregulation of the initial reprogramming genes GDF3, NODAL, $D P P A 3, D N M T 3 B$ and GAL [10] (Figure 2A, 2B). SOX2 was not significantly upregulated in TCam-2- $\triangle \mathrm{SOX} 2$ clones compared to TCam-2 in vitro. Other pluripotency factors were either slightly down- (OCT3/4, PRDM14) or upregulated (ZFP42) or remained unchanged (ZIC3) compared to in vitro cultivated TCam-2, but expression intenstities were clearly different to xenografted TCam-2 or 2102EP. In TCam-2- $\triangle \mathrm{SOX} 2$ clones, expression of seminoma markers SOX17, PRAME, TFAP2C, LIN28 and $P R D M 1$ was slightly downregulated or remained unchanged compared to the TCam-2 in vitro cells. Initially during the seminoma to EC transition, BMP signaling is inhibited, indicated by strong downregulation of $I D 1 / 3$ [10]. At late stages of the reprogramming, BMP signaling is re-activated by upregulating BMP4 [10]. Additionally, expression of $I D 1 / 3$ recovers to a level lower than in parental TCam-2 cells [10]. Inhibition of BMP signaling leads to activation of NODAL signaling [10]. In TCam-2- $\Delta$ SOX2 clones, 1 and 6 week(s) after xenografting we found downregulation of $I D I / 3$ to a level comparable to xenografted TCam-2/2102EP (Data S1C; Figure 2A, 2B). This suggests that during in vivo growth of TCam-2- $\triangle \mathrm{SOX} 2$ clones, like during reprogramming of parental TCam-2, BMP signaling is inhibited and remains constantly low compared to parental TCam-2 cells [10]. Nevertheless, NODAL, LEFTY1 and LEFTY2 were not significantly upregulated, indicating inactive NODAL signaling and suggesting that NODAL signaling is regulated by SOX2.

IHC staining confirmed absence of SOX2 and expression of SOX17, OCT3/4 and TFAP2C in all xenografted TCam-2- $\triangle \mathrm{SOX} 2$ clones (Figure 3 ). 
A
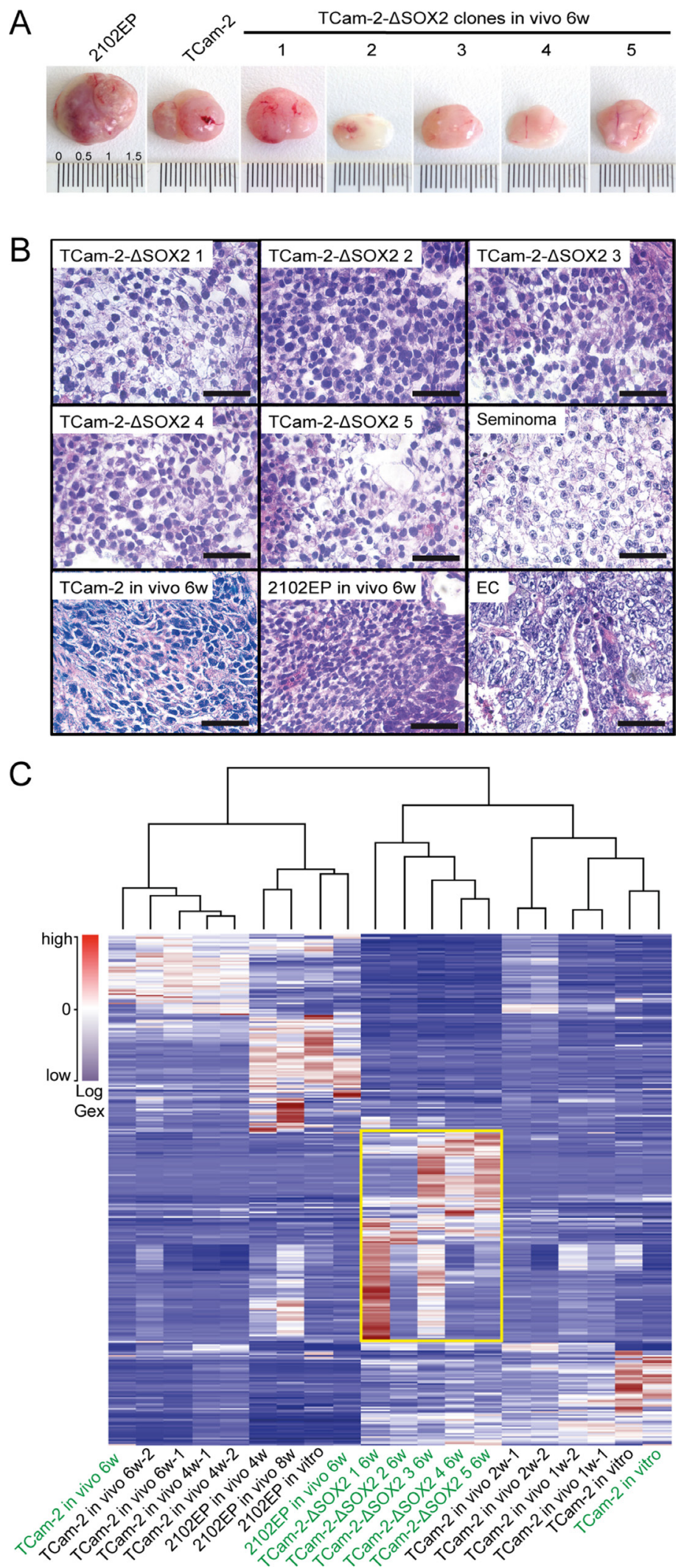

Figure 1: (A) Macroscopical appearance of tumors from xenografted TCam-2, 2102EP and TCam-2- $\Delta$ SOX2 clones. (B) HE staining of tumor tissues from xenografted TCam-2- $\Delta$ SOX2 clones, parental TCam-2 and 2102EP. A seminoma and an EC tissue were stained as controls. Scale bars: $50 \mu \mathrm{m}$. (C) Meta-analysis of expression microarray data of indicated samples including unsupervised hierarchical clustering. Samples labelled in green were generated for this study, samples in black are part of a previous publication [10] and re-analyzed in context of this study. The yellow box highlights genes differentially expressed between TCam-2- $\Delta \mathrm{SOX} 2$ clones and the other samples. 


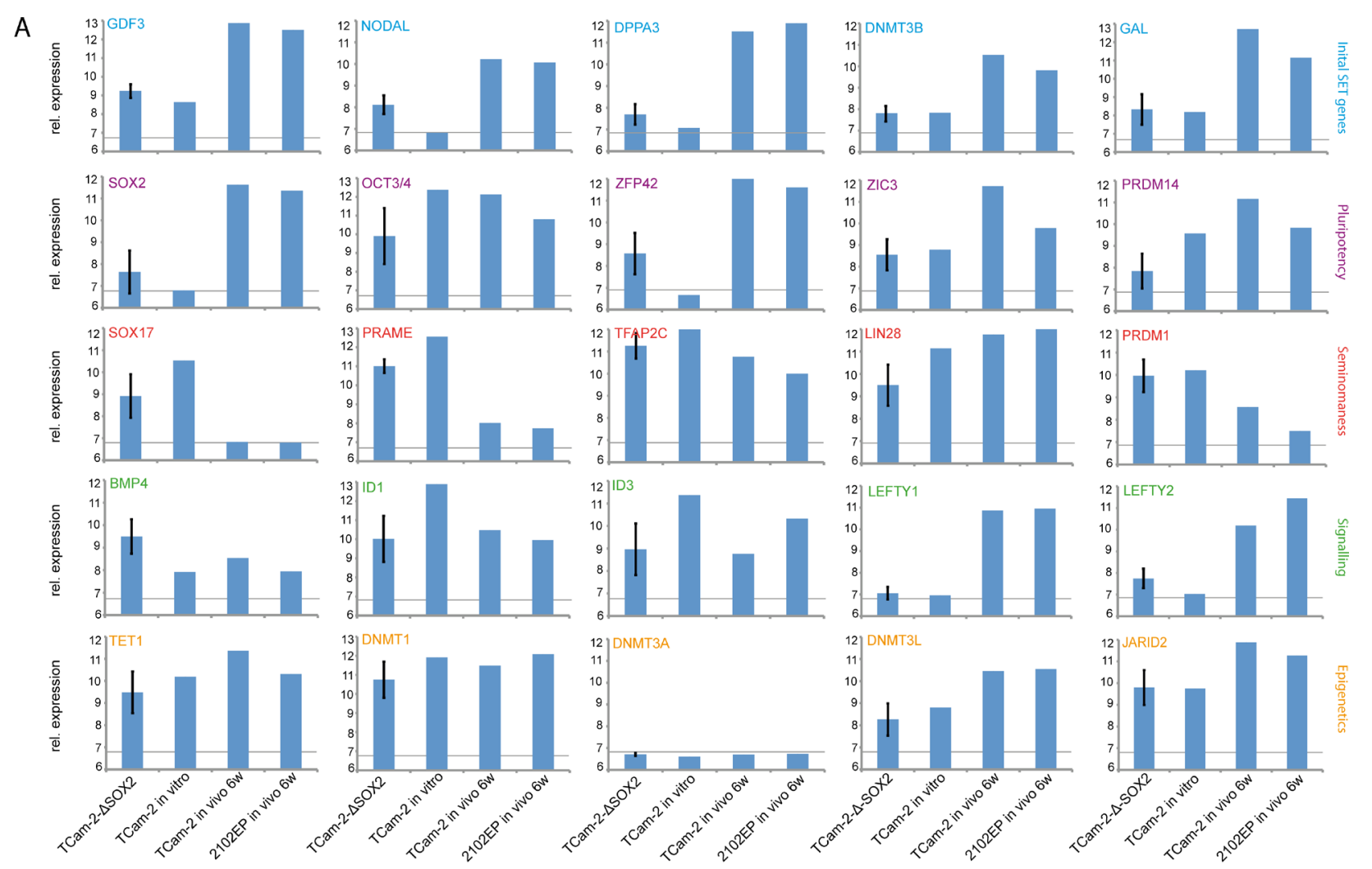

B
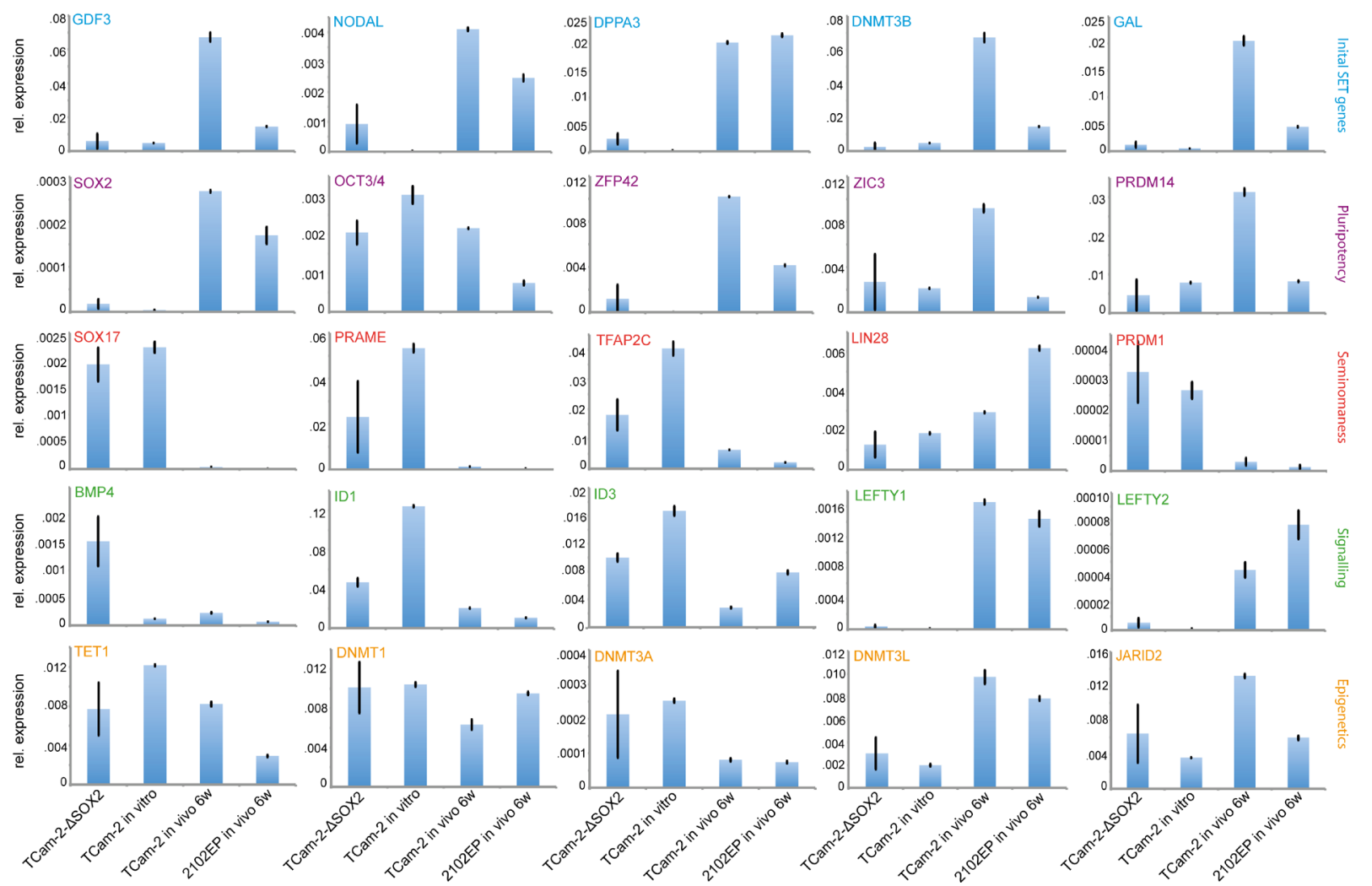

Figure 2: (A, B) Expression microarray (A) and qRT-PCR (B) data of indicated genes related to the in vivo reprogramming, pluripotency, seminoma-ness, signaling pathways and epigentics. For TCam-2- $\Delta \mathrm{SOX} 2$ clones, expression data of five clones was averaged. Standard deviation is indicated above each bar. Genes showing expression values below the grey line in (A) were considered as not expressed $\left(\log _{2} 6.8\right)$. 
Furthermore, IHC demonstrated nuclear localization of PRDM1, which is a hallmark of GCNIS/seminomas, while in ECs PRDM1 is located in the cytoplasm [11] (Figure 3). In murine PGCs, nuclear Prdm1 establishes in concert with Prmt5 a symmetric dimethylation of arginine 3 on histones $\mathrm{H} 2 \mathrm{~A}$ and $\mathrm{H} 4$ (H2A/H4R3me2s), which suppresses somatic differentiation programs [12, 13]. Accordingly, H4R3me2s was strongly detectable in TCam-2- $\triangle \mathrm{SOX} 2$ clones, but not in 2102EP cells (Figure 3). This suggests that in PRDM1/ H4R3me2s positive TCam-2- $\triangle \mathrm{SOX} 2$ cells, somatic differentiation is epigenetically blocked, contributing to maintenance of a seminoma-like cell fate. A strong Ki67 signal indicated a high proliferative activity of the TCam2- $\triangle \mathrm{SOX} 2$ tumor cells (Figure 3 ).

Our data suggested that NODAL signaling might be regulated by SOX2. Thus, we analyzed 2102EP cells as a proxy for an EC by chromatin-immunoprecipitation (ChIP) using a SOX2 antibody followed by qPCRanalysis. We detected enrichment of SOX2 at the promotors of the NODAL signaling key factors LEFTY1/ LEFTY2 and CRIPTO, which contain SOX2 binding sites (Figure 4) [14]. NODAL itself was not enriched over input control (Figure 4). As positive controls, we analyzed the promoters of $S O X 2, N A N O G$ and $O C T 3 / 4$ and confirmed enrichment of SOX2 at these elements (Figure 4) [15-18]. All genes analyzed are also expressed in 2102EP/ EC cells $[10,19]$. As a negative control, the promoter of the RPL30 Gene was analyzed, where we did not detect enrichment of SOX2 (Figure 4). Thus, it is highly likely that during in vivo growth of TCam-2- $\triangle \mathrm{SOX} 2$ clones NODAL signaling remains inactive, because induction of the essential NODAL co-factor CRIPTO and LEFTY $1 / 2$ and subsequently establishment of the NODAL signaling loop requires $\mathrm{SOX} 2$.

We asked, if other factors that contain a SOX2 binding site and are involved in reprogramming of TCam-2 to an EC might be bound and regulated by SOX2. Again, we analyzed 2102EP cells as a proxy for an EC by SOX2ChIP and found enrichment of SOX2 at corresponding binding sites within the promoters of DNMT3B, PRDM14, JARID2 and DPPA4 (Figure 4). The fact that these factors are strongly upregulated during the seminoma to EC transition of TCam-2, suggests that SOX2 induces expression of these genes [10].

The seminoma to EC transition is accompanied by upregulation of the de novo DNA methyltransferase $D N M T 3 B$, resulting in a strong increase in DNA methylation levels $[8,10]$. In TCam-2- $\triangle \mathrm{SOX} 2$ cells, $D N M T 3 B$ is not upregulated and the expression levels of other epigenetic regulators (TET1, DNMT1, DNMT3A, $D N M T 3 L, J A R I D 2)$ are highly comparable to in vitro cultivated TCam-2 (Figure 2A, 2B). Thus, depletion of $S O X 2$ prevents the induction of DNMT3B and other epigenetic factors. In line, global DNA methylation levels do not increase in TCam-2- $\triangle \mathrm{SOX} 2$ clones, but in xenografted TCam-2 cells that adapted to an EC-like fate and 2102EP cells (Figure 5A).

In summary, in SOX2 depleted clones expression of seminoma markers is maintained and the initial reprogramming markers, pluripotency genes and epigenetic factors are not induced. Additionally, although BMP signaling seems to decrease and recover during in vivo growth of the TCam-2- $\triangle \mathrm{SOX} 2$ clones, NODAL signaling is not activated, suggesting that SOX2 controls activation of NODAL signaling. Further, expression of epigenetic factors, including de novo DNA methyltransferases and DNA methylation levels remain at the level of TCam-2. Thus, the data demonstrate that inhibition of BMP signaling does not depend on SOX2. Interestingly, the lack of NODAL activation strongly suggests that SOX2 is essential for this step. Hence, SOX2 acts downstream of BMP signaling and upstream of the NODAL cascade.

Although the vast majority of cells stained positive for the seminoma markers SOX17, OCT3/4 and TFAP2C, small areas displayed absence of staining of these pluripotency- and seminoma-related genes, pointing at a heterogeneity of the tumor tissues (Figure 3, red arrows). We hypothesize that this subpopulation represents a differentiated population.

In a previous study, we demonstrated that TCam-2 cells directly differentiate in vitro into a cell type resembling a mixed non-seminoma, when being cultivated in fibroblast-conditioned medium supplemented with FGF4/heparin [20]. During this differentiation, an EC-intermediate step is skipped (no upregulation of SOX2) and a strong upregulation of germ layer differentiation markers, like $A F P$ (also a yolk-sac tumor marker) and HAND1 was observed [20]. SOX17 expression was downregulated [20]. Morphologically, the cells presented as large roundish cells with a big nucleus [20]. Additionally, the cells showed features of trophectodermal/choriocarcinoma-like cells, i. e. presence of multinucleiated cells and upregulation of GATA2, GATA6, TEAD1 and TEAD4 [20]. Pluripotency and seminoma markers like NANOG, OCT3/4, LIN28, TFAP2C, $c K I T, D 2-40$ and PRDM1 were downregulated [20]. Downregulation of PRDM1 was accompanied by reduced levels of $\mathrm{H} 2 \mathrm{a} / \mathrm{H} 4 \mathrm{R} 3 \mathrm{me} 2 \mathrm{~s}$, allowing for differentiation [20].

We repeated this in vitro differentiation experiment and included the TCam-2- $\triangle \mathrm{SOX} 2$ clones (Figure S2E). Under in vitro differentiating conditons both, the parental and SOX2-deficient TCam-2 cells downregulated pluripotency/seminoma markers OCT3/4, TFAP2C, PRDM14 and PRAME, while SOX2 and DNMT3B expression remained low (Figure S2F). In contrast, expression of the trophoblast stem cell marker EOMES and germ layer differentiation markers $A F P, H A N D 1$, $F O X A 2$ (endoderm) and $P A X 6$ (ectoderm) were strongly upregulated (Figure S2F). SOX17 remained expressed, but to a level considerably lower than under non- 


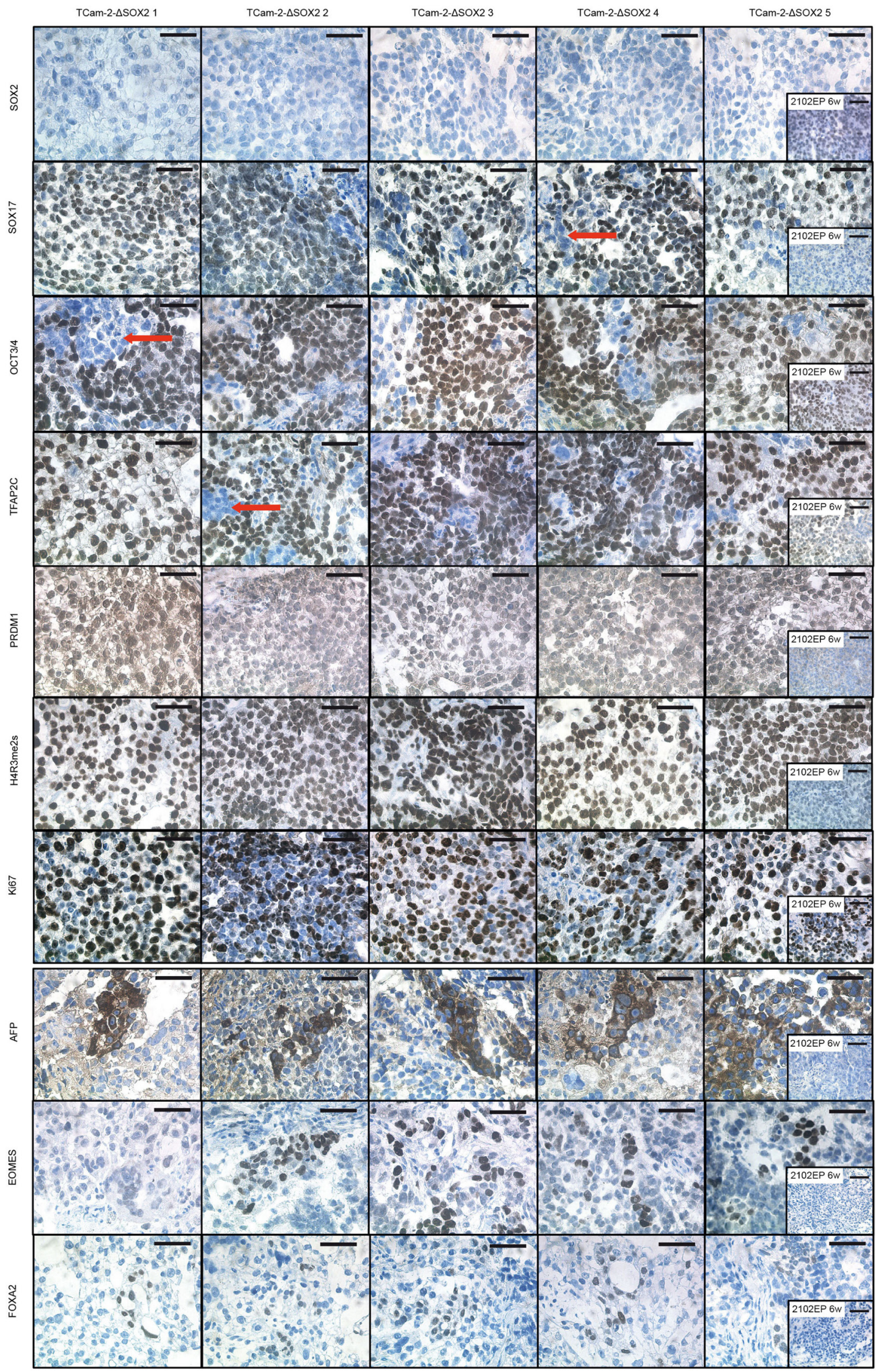

Figure 3: IHC staining of indicated proteins in TCam-2- $\triangle$ SOX2 clones. Tumor tissues from xenografted 2102EP cells were stained as control. Scale bars: $50 \mu \mathrm{m}$. 
differentiating conditions (Figure S2F) [20]. Similar to the in vivo growth of TCam-2- $\triangle \mathrm{SOX} 2$ cells, NODAL, but not LEFTY1/LEFTY2 or CRIPTO was upregulated under differentiating conditions, further strengthening the notion that SOX2 is necessary to build up a functional NODAL signaling loop (Figure S2F). In summary, the deficiency of SOX2 does not impair the in vitro differentiation process of TCam-2 cells into a cell type resembling a mixed nonseminoma, further demonstrating that no EC intermediate state is necessary.

We screened the microarray data of TCam-2- $\Delta \mathrm{SOX} 2$ for deregulations in gene expression indicative for a differentiation process similar to the in vitro differentiation of TCam-2. From all genes differentially expressed between TCam2- $\triangle \mathrm{SOX} 2$ clones $(6 \mathrm{w})$ and TCam-2 in vitro, we found 124 probes coding for 112 annotated genes significantly (fold change $\geq \log _{2} 1.5$ ) upregulated and 500 probes (437 annotated genes) downregulated (Data S1 B). In the TCam-2- $\mathrm{SOXX} 2$ clones, we found very similar results as during the in vitro differentiation of TCam-2, i. e. upregulation of endodermal ( $A F P$, FOXA2, CDX1), mesodermal (HAND1, PRRX1, PDGFRA, GJA1, PITX2, CXXC5, DKK1, AIF1, MSX1, GPC3, DACT3, HOXC8) and ectodermal (SOX11, DLK1, $B E X 1)$ differentiation markers as well as downregulation of pluripotency factors OCT3/4 and LIN28 (Data S1B). Additionally, the trophoblast stem cell marker EOMES and syncytiotrophoblast-associated chorionic gonadotropins $C G B 1, C G B 5$, CGB8 were upregulated in TCam-2$\triangle \mathrm{SOX} 2$ clones compared to TCam-2 in vitro (Data $\mathrm{S} 1 \mathrm{~B}$ ).
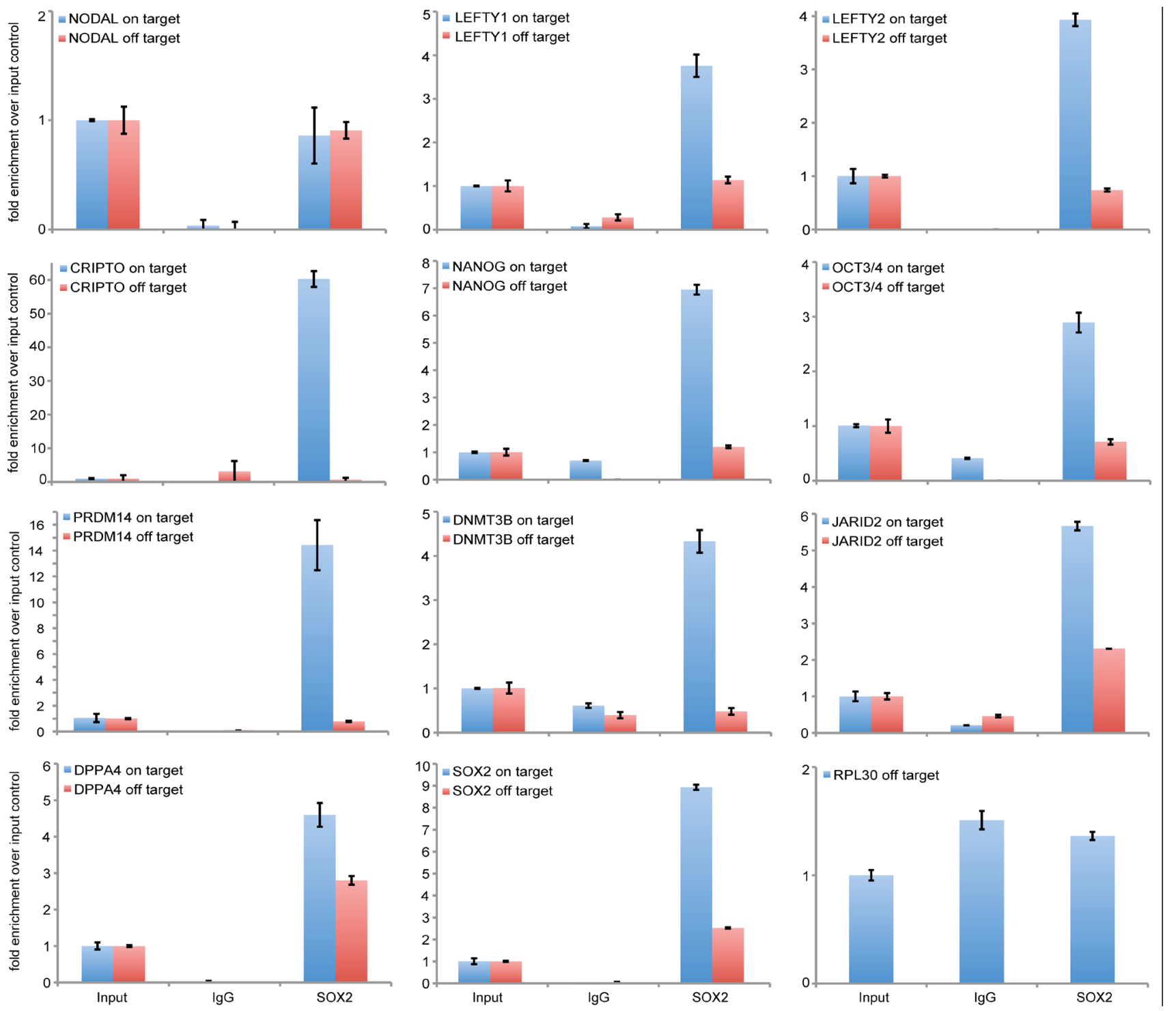

Figure 4: ChIP followed by qPCR-analysis of SOX2-enrichment at the SOX2 binding sites within indicated genes. $3 \%$ input chromatin was used for normalization and an IgG antibody was used as negative ChIP control. In qPCR, oligonucleotides were used to amplify a PCR-fragment around the SOX2 binding site (on target) and a PCR-fragment within the same gene, but without a SOX2 binding site (off target). 
By IHC, we confirmed upregulation of AFP, FOXA2 and EOMES in a subset of cells morphologically appearing differentiated within TCam-2- $\triangle \mathrm{SOX} 2$ tumor tissues (Figure 3). Additionally, qRT-PCR analysis validated upregulation of $A F P$ and $H A N D 1$ in TCam-2- $\triangle \mathrm{SOX} 2$ (Figure S3A).

Within the OCT3/4/SOX17/TFAP2C negative subpopulation, very large roundish cells with a big nucleus (green arrows) and multinucleated cells (red arrows) were found (Figure S3 B). Furthermore, PRDM1 was excluded from the nucleus in these cells (Figure S3 B, blue arrows). We postulate that the small subpopulation of OCT3/4/ SOX17/TFAP2C negative cells is highly similar to in vitro differentiated TCam- 2 and thus resembles a mixed nonseminoma in vivo.

We asked, if first signs of a differentiation process into a mixed non-seminoma can be found already one week after xenografting. Therefore, we compared all genes deregulated in TCam-2- $\Delta$ SOX 2 clones 6 weeks after xenografting to genes deregulated one week after xenografting (fold change $\geq \log _{2} 2$ ). We found only 14 genes commonly upregulated and 11 downregulated (Data S1 C). The upregulated genes were not indicative for a differentiation process and from the downregulated genes no loss of seminona-like fate could be concluded. Although, ID 3 was among the set of downregulated genes, pointing at inhibited BMP signaling as already discussed. In conclusion, differentiation into a mixed non-seminoma is initiated later than one week after xenografting.

To support our hypothesis of a mixed non-seminomalike differentiation process, we compared the deregulations found in the TCam-2- $\triangle \mathrm{SOX} 2$ clones to differences in gene expression between non-seminoma and seminoma tissues (fold change $\geq \log _{2} 1.5$ ) gained from microarray data published previously $[10,21]$ (Data S1 D). From the 122 genes found to be upregulated in TCam-2- $\Delta \mathrm{SOX} 2$ clones versus TCam-2 in vitro, 42 genes were also upregulated in non-seminomas versus seminomas (Figure 5B, Data S1D). Among them, differentiation markers like AFP, ALB, DKK1, FST, GJA1, PITX2, GPC3, CGA and several apolipoproteins and fibrinogens (Figure $5 \mathrm{~B}$ ). This suggests that the cluster of genes upregulated during in vivo differentiation of TCam-2- $\Delta \mathrm{SOX} 2$ cells reflects differences in expression between non-seminomas and seminomas, confirming our postulated non-seminomatous differentiation process.

In TCam-2- $\triangle \mathrm{SOX} 2$ cells, we found a strong upregulation of $F O X A 2$, a pioneer factor able to open compacted chromatin and regulator of expression in differentiated tissues and during embryonic development [22-24]. FOXA2 has been described as a transcriptional activator for AFP and albumin (ALB) and regulator of lipid metabolism and fibrinogens [25-27]. Furthermore, FOXA2 is involved in development of endodermalderived organs like the liver, pancreas and lungs $[28,29]$. In TCam-2- $\triangle$ SOX 2 clones, we found a strong upregulation of FOXA2 target genes $A F P$ and $A L B$ (Data S1B). Additionally, lipid metabolism associated apolipoproteins (APOA1, APOA2, APOB, APOC1, APOE, APOH, $A P O M$ ), fibrinogens and related factors ( $F G A, F G B, F G G$, $F G L 1, F L R T 3)$ as well as several endodermal factors (HPX, CDX1) were upregulated (Data S1B). STRING analysis of all genes upregulated in TCam-2- $\triangle \mathrm{SOX} 2$ clones versus TCam-2 in vitro predicted interaction of FOXA2 with many of these genes (Data S1E). Among the genes commonly expressed in TCam-2- $\Delta$ SOX2 clones (vs. TCam-2 in vitro) and in non-seminomas (vs. seminomas) are several FOXA2 targets (Figure 5B, green labeled genes; Data S1E). Thus, in TCam-2- $\Delta$ SOX2 clones FOXA2 might be an important driver of somatic differentiation.

\section{DISCUSSION}

In this study, we generated SOX2-deficient TCam-2 cells by the CRISPR/Cas9 technique and xenografted these cells into the flank of nude mice. Molecular analyses of the tumors demonstrated a seminoma-like morphology and gene expression profile, suggesting that SOX2 is essential for induction of an EC-like cell fate. In TCam-2$\triangle \mathrm{SOX} 2$ clones, BMP signaling related molecules $I D 1$ and ID3 were downregulated, which is indicative for reduced BMP signaling activity. However, activation of NODAL (early during reprogramming of TCam-2) and WNT (late during reprogramming) signaling and upregulation of pluripotency and EC markers $G D F 3, D P P A 3, D N M T 3 B$, ZIC3, PRDM14 failed to occur [10]. Thus, SOX2 appears to be downstream of BMP signaling but upstream of NODAL activation. Our ChIP analysis in 2102EP suggested that SOX2 regulates expression of LEFTY1/ LEFTY2 and CRIPTO, allowing for establishment of the NODAL signaling loop. In vitro treatment of TCam-2 cells (SOX2 negative) with recombinant NODAL was not sufficient to activate NODAL signaling [10]. So, expression of SOX2 is a prerequisite for activation of NODAL signaling. Furthermore, our ChIP experiments demonstrated binding of SOX2 to key factors of pluripotency and ECs (SOX2, DPPA3, DNMT3B, $P R D M 14, G D F 3)$. In conclusion, SOX2 is an essential factor in acquiring the EC-like cell fate from GCNIS or seminoma.

Most importantly, the majority of TCam-2- $\Delta \mathrm{SOX} 2$ cells maintains a seminoma-like cell fate for at least 6 weeks in vivo, indicated by a typical seminoma-like morphology and expression of seminoma markers SOX17, PRAME, TFAP2C and PRDM1 (nuclear). Nevertheless, we observed an OCT3/4, SOX17 and TFAP2C negative subpopulation showing cytoplasmic PRDM1 and lack of H4R3me2s. Additionally, microarray analyses revealed upregulation of markers indicative for differentiation into all three germ layers, like AFP, HAND1 and CDX1. This expression profile 
is highly similar to in vitro differentiated TCam-2 cells that resemble a mixed non-seminoma [20]. There, the differentiated cells also upregulated $A F P$ and $H A N D 1$ and showed nuclear exclusion of PRDM1. AFP is a strong marker for the non-seminomatous GCC entity yolk-sac tumor. Additionally, cells appeared morphologically as big roundish cells with a big nucleus - a morphology also found in the OCT3/4/SOX17/TFAP2C negative subpopulation of TCam-2- $\triangle \mathrm{SOX} 2$ tumors. Furthermore, in vitro differentiated TCam-2 gained features of trophoblast cells, like upregulation of TEAD4 and formation of syncytiotrophoblastic multinucleated cells. We also found multinucleated cells within the differentiated subpopulation of in vivo grown TCam-2$\triangle \mathrm{SOX} 2$ cells as well as upregulation of the trophoblast stem cell marker EOMES. So, the OCT3/4/SOX17/ TFAP2C negative subpopulation cleary recapitulates the in vitro differentiation process of TCam-2 cells into a mixed non-seminoma in vivo. As in vitro, an EC intermediate is skipped. Thus, we showed for the first time that TCam-2 cells are able to directly differentiate into a mixed non-seminoma in vivo. This development of a mixed non-seminomatous tumor does not require a SOX2-positive EC intermediate.

How is the differentiation process initiated? In murine ESCs, SOX2 promotes pluripotency, while SOX17 drives differentiation into endodermal lineage. Both, SOX2 and SOX17 are able to partner with OCT3/4, but different binding motifs are occupied, regulating expression of pluripotency- and self-renewalassociated factors (SOX2/OCT3/4) or differentiationrelated genes (SOX17/OCT3/4). In human seminomas/ TCam-2 cells, SOX17 and OCT3/4 are expressed, but endodermal differentiation programs are suppressed. We demonstrated that in TCam-2, SOX17 coimmunoprecipitates with OCT3/4 and is able to bind to the NANOG promotor containing a SOX2/OCT3/4 binding motif (Figure 5D, 5E). Thus, in undifferentiated seminomas it is highly likely that SOX17 is redundant to SOX2 and promotes expression of pluripotency factors in combinaton with OCT3/4, thereby maintaining a GCNIS/ seminoma-like cell fate.

During reprogramming of TCam-2, SOX17 is replaced by SOX2, leading to acquisition of an EC-like fate. In TCam-2- $\triangle \mathrm{SOX} 2$ cells, the switch from SOX17 to SOX2 is not possible and prolonged expression of SOX17/ OCT3/4 contributes to maintenance of a seminoma fate.

In the differentiated subpopulation of in vivo grown TCam-2- $\triangle \mathrm{SOX} 2$ cells, we found a strong upregulation of FOXA2, a pioneer factor, which is able to open compacted chromatin and regulate gene expression in differentiated tissues and during embryonic development [22, 23, 24]. Additionally, several FOXA2 target genes were upregulated in TCam2- $\triangle \mathrm{SOX} 2$ clones. Thus, FOXA2 might be an important factor in promoting differentiation of the TCam-2$\triangle \mathrm{SOX} 2$ cells into a mixed non-seminoma. In line, the FOXA2 protein was detectable only in OCT3/4/SOX17/ TFAP2C negative cells, which appear morphologically as differentiated.

Interestingly, the FOXA2-driven differentiation seems to be independent of SOX17, since the SOX17 protein is not detectable in differentiated TCam-2$\triangle \mathrm{SOX} 2$ subpopulation. Thus, in contrast to the murine system, SOX17 presumably is not involved in the differentiation of human seminomas into a cell type resembling a mixed non-seminoma.

Together with the protein arginine methyltransferase PRMT5, nuclear PRDM1 dimethylates arginine 3 on the histones H2A and H4. By this epigenetic mechanism, somatic differentiation programs are suppressed in murine PGCs [12, 13]. Nuclear PRDM1 and the H2A/H4R3me2s was also found in human seminomas, but not in ECs [11]. During in vitro differention of TCam-2 into a mixed non-seminoma and during the in vivo reprogramming, PRDM1 is downregulated and excluded from the nucleus $[10,20,30]$. Thus, in TCam-2- $\triangle \mathrm{SOX} 2$ clones, nuclear PRDM1 might be responsible for maintenance of $\mathrm{H} 2 \mathrm{~A} / \mathrm{H} 4 \mathrm{R} 3 \mathrm{me} 2 \mathrm{~s}$ and suppression of somatic differentiation. In contrast, in the OCT3/4/SOX17/ TFAP2C negative subpopulation nuclear exclusion of PRDM1 leads to reduced H2A/H4R3me2s levels, putatively enabling differentiation.

In summary, TCam-2 grow as seminoma-like in the testis and transit into an EC-like fate when being xenografted into the somatic microenvironment of the murine flank or brain (Figure 6A) [8, 10, 30]. Additionally, TCam-2 can be forced to differentiate into a mixed non-seminoma upon cultivation of murine fibroblast conditioned medium supplemented with FGF4 (Figure 6A) [20]. TCam-2- $\triangle \mathrm{SOX} 2$ maintain a seminoma-like fate in vivo for at least 6 weeks, but initiation of differentiation in a subpopulation cannot be prevented (Figure 6A). This differentiation is highly similar to the in vitro differentiation into a mixed nonseminoma. Recently, a study found high intratumoral heterogeneity in GCC tissues from about 615 patients [31]. There, patients suffering from a mixed yolk-sacseminoma tumor had the poorest clinical outcome [31]. In our study, strong upregulation of AFP in the OCT3/4 negative TCam-2- $\triangle \mathrm{SOX} 2$ subpopulation is indicative for development of a yolk-sac tumor. Thus, 6 weeks after xenografting a yolk-sac-seminoma tumor component is present. So, upon contact with a somatic microenvironment, seminomas may develop into more aggressive yolk-sac-seminoma-like tumors that need different therapeutic strategies as classical seminomas. $\mathrm{Tu}$ et al. propose that an integrated or multimodal therapy may be effective at addressing intratumoral 
heterogeneity and treating distinct subtypes as well as a potentially lethal phenotype of non-seminomatous GCCs [31]. Xenografting of TCam-2- $\triangle \mathrm{SOX} 2$ cells provides a useful model to adress these issues regarding mixed yolk-sac-seminoma-like tumors.

We have shown evidence that upon xenografting of TCam-2 cells into the flank SOX17 is replaced by SOX2 [8] [10]. Based on our previous published data and this study, we propose that the somatic microenvironment inhibits BMP signaling, leading to upregulation of SOX2, which partners with OCT3/4. Together, SOX2 and OCT3/4 drive acquisition of an EC-like fate by contributing to establishment of the NODAL signaling loop (LEFTY1/2, CRIPTO) and regulating pluripotency- (SOX2,
OCT3/4, NANOG, PRDM14, DPPA4) and epigenetic reprogramming-factors (DNMT3B, JARID2) (Figure 6B, upper panel; C). In seminoma-like TCam-2- $\Delta$ SOX2 cells SOX17 expression is maintained and presumably partners with OCT3/4 to bind the canonical motif, triggering expression of PGC-, GCNIS- and seminoma-related genes, allowing keeping up the seminoma-like cell fate (Figure 6B, middle panel). Nevertheless, differentiation into a mixed non-seminoma is not blocked in TCam-2$\triangle \mathrm{SOX} 2$ cells. We hypothesize that in this OCT3/4/SOX17 negative subpopulation the pioneer factor FOXA2 initiates differentiation into endodermal-, mesodermal- and ectodermal-lineage as well as trophectoderm (Figure 6B, lower panel).
A

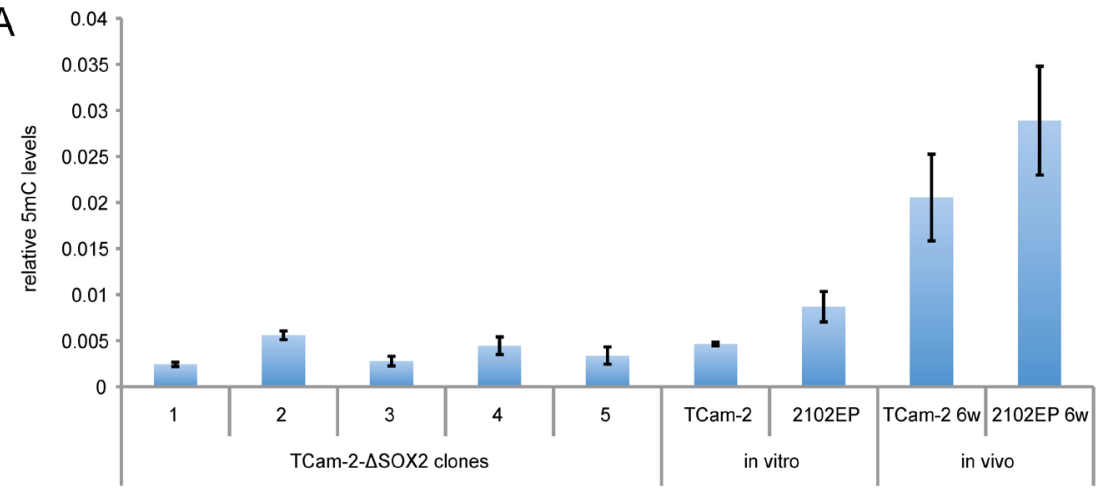

C

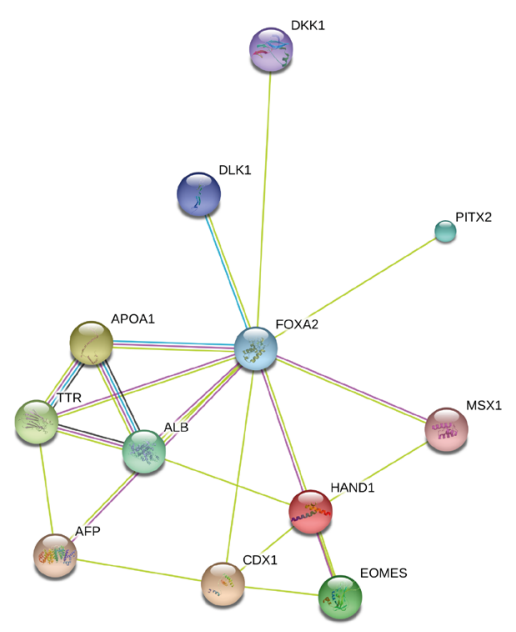

D

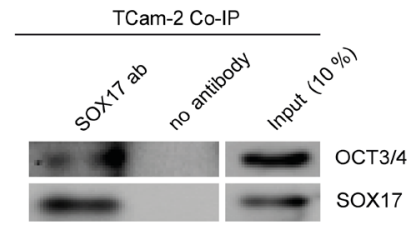

B

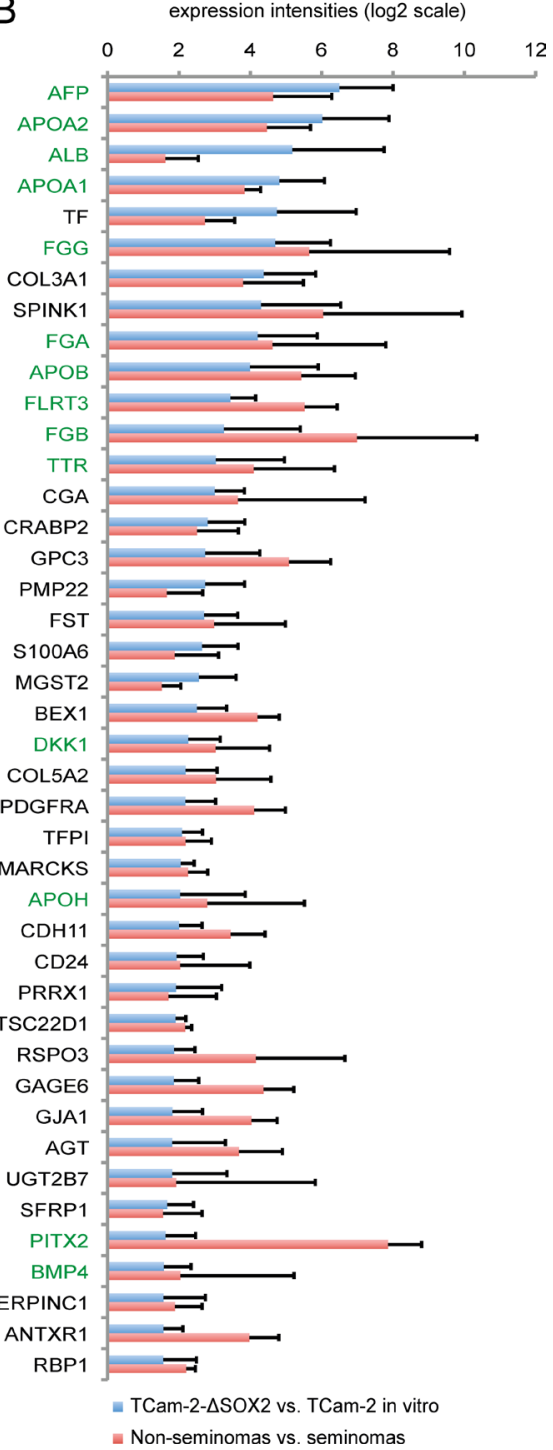

Figure 5: (A) Quantification of $5 \mathrm{mC}$ levels in tumors from xenografted TCam-2- $\triangle \mathrm{SOX} 2$ clones, TCam-2 and $2102 \mathrm{EP}$ cells. In vitro cultivated TCam-2 and 2102EP served as controls. (B) Commonly expressed genes in TCam-2- $\triangle \mathrm{SOX} 2$ (vs. TCam-2 in vitro) and nonseminomas $(n=7)$ (vs. seminomas $(n=4)$ ) based on microarray data. Green labeled genes are related to FOXA2. (C) STRING analysis of FOXA2 interacting genes. (D) Co-IP experiment in TCam-2 demonstrating binding of SOX17 to OCT3/4. (E) ChIP experiment in TCam-2, demonstrating binding of SOX17 to the SOX2 / OCT3/4 binding motif within the NANOG promoter. 
A

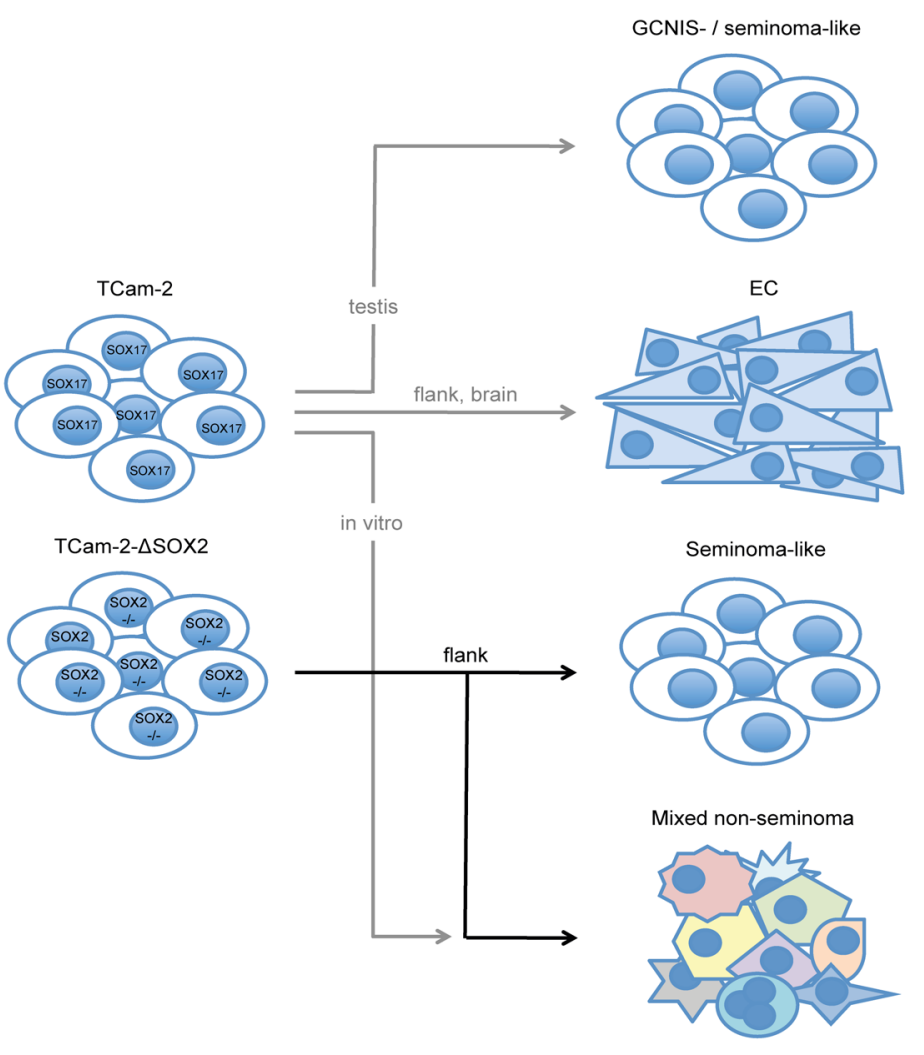

B

B

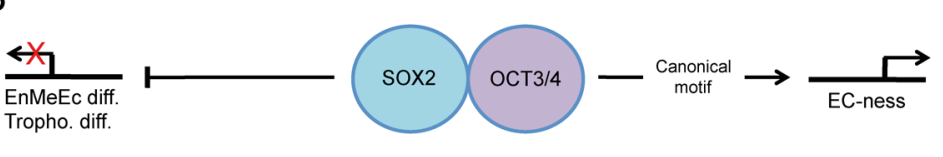

TCam-2 in vitro / TCam-2- $\triangle \mathrm{SOX} 2$ in vivo (undifferentiated population)

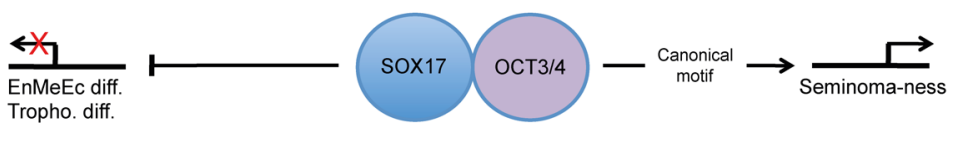

TCam-2- $\triangle \mathrm{SOX} 2$ in vivo (differentiated population)

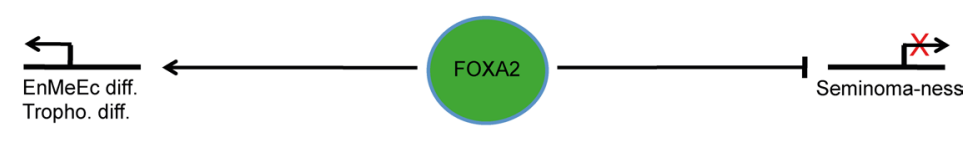

C

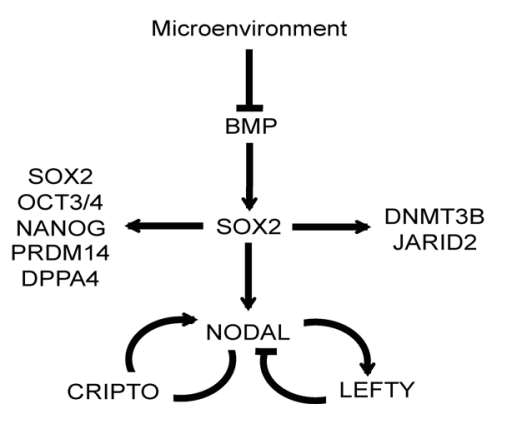

Figure 6: (A) Model of the differentiation abilities of TCam-2 and TCam-2- $\Delta$ SOX2 cells in vitro and in vivo. (B) Molecular mechanisms of promoting EC- or seminoma-ness and differentiation into endodermal-, mesodermal- and ectodermal-lineage (EnMeEc diff.) and trophectoderm (Troph. diff.). (C) Detailed mechanism of the role of SOX2 in reprogramming of TCam-2 to an EC-like state in vivo. Models are based on the results of this study and $[8,10,20,30]$. 


\section{MATERIAL AND METHODS}

\section{Ethics statement}

All animal experiments were conducted according to the German law of animal protection and in agreement with the approval of the local institutional animal care committees (Landesamt für Natur, Umwelt und Verbraucherschutz, North Rhine-Westphalia (approval ID: AZ-84-02.04.2013-A430). The experiments were conducted in accordance with the International Guiding Principles for Biomedical Research Involving Animals as announced by the Society for the Study of Reproduction.

\section{Cell culture}

GCC cell lines utilized in this study were cultivated as described previously [10]. Briefly, TCam-2 cells (provided by Dr. Janet Shipley, Institute of Cancer Research, Sutton, UK) were grown in RPMI, 2102EP and NCCIT (both from Prof. Dr. Leendert Looijenga, Erasmus MC, Daniel den Hoed Cancer Center, Josephine Nefkens Institute, Rotterdam, NL) in DMEM. In vitro differentiation of TCam-2 and TCam-2- $\triangle \mathrm{SOX} 2$ was induced as published [20]. Briefly, $1 \times 10^{4}$ cells were cultivated for 10 days in murine embryonic fibroblast conditioned medium supplemented with FGF $(25 \mathrm{ng} / \mathrm{ml})$ and Heparin $(25 \mathrm{ng} / \mathrm{ml}$ ) (both from R\&D Systems, Wiesbaden, Germany). The medium was exchanged every two days.

\section{Generation of SOX2-deficient cells by CRISPR/ Cas9}

To delete the $S O X 2$ gene in TCam-2 cells, we transfected TCam-2 cells simultaneously with the pX330 vector encoding for three different guide RNAs (gRNA) directed towards the SOX2 coding region using FuGeneHD (Promega, Mannheim, Germany) (transfection ratio $5: 1 ; \mu 1$ FuGeneHD : $\mu \mathrm{g}$ pX330) (Figure $\mathrm{S} 1 \mathrm{~A}$, Table 2) and a GFP-coding plasmid (transfected at a 10× lower concentration compared to the pX330 vector). Two days post transfection, GFP-positive clones, which presumably have taken up the pX330 plasmids were identified, manually picked and clonally expanded.

\section{DNA, RNA and protein isolation}

DNA, RNA and proteins were isolated as described previously [10]. DNA was isolated by phenol/chloroform/ isoamylalcohol, RNA by TRIzol and proteins by RIPA buffer.

\section{Quantitative RT-PCR}

Quantitative RT-PCR (qRT-PCR) was performed as described previously [10]. At the end of each PCR run, a melting point analysis was performed. GAPDH was used as housekeeping gene and for data normalization. In each qRT-PCR, the TCam-2- $\Delta$ SOX2 clones were analyzed in 5 biological replicates and each replicate was analyzed in 3 technical replicates.

\section{Immunohistochemistry}

Immunohistochemistry (IHC) was performed as published previously [10]. Tumor tissues were dissected, fixed in $4 \%$ formalin overnight and processed in paraffin wax. Signal detection was performed semiautomatically in the Autostainer $480 \mathrm{~S}$ (Medac, Hamburg, Germany). Nuclei were stained by hematoxylin. See Table S1 for antibody details and dilution ratios. For IHC, 5 tumor tissues from TCam-2- $\triangle \mathrm{SOX} 2$ clones and 1 tumor tissue from $2102 \mathrm{EP}$ cells as control was analyzed.

\section{Co-immunoprecipitation (Co-IP)}

Co-IP was performed using Dynabeads Protein G beads (Life Technologies, Darmstadt, Germany) according to the manual. For each Co-IP reaction, $1.5 \mathrm{mg}$ Dynabeads and $10 \mu \mathrm{g}$ IP-antibody were used. Antibody binding was performed at room temperature (RT) for 40 minutes (min). Immunoprecipitation of target antigen was performed at RT for 2 hours (h). $200 \mu \mathrm{g}$ of total protein lysate were used. $10 \%$ of the whole protein lysate $(20 \mu \mathrm{g})$ were used as input control in western blotting. For elution, the Dynabead-protein-complexes were re-suspended in $20 \mu 1$ western blot loading buffer $(1 \times$ Lämmli buffer in aqua. dest.) and incubated at $95^{\circ} \mathrm{C}$ for $5 \mathrm{~min}$. Afterwards, Dynabeads were removed by a magnet and eluted samples were analyzed by western blotting. See Table S1 for antibody details.

\section{Chromatin-immunoprecipitation (ChIP)}

For ChIP, the 'SimpleChIP Enzymatic Chromatin IP Kit (magnetic Beads)' (Cell Signaling Technology, via NEB, Frankfurt a. M., Germany) was used according to the protocol. Briefly, $1 \times 10^{7}$ cells were cross-linked by formaldehyde and chromatin was sheared enzymatically. For each ChIP, $30 \mu \mathrm{g}$ Dynabeads and $5 \mu \mathrm{g}$ of the SOX2 antibody were used. As control, an antibody against rabbit $\operatorname{IgG}$ was included. $2 \%$ of sheared chromatin was used as input control. Antibody binding to target complexes was performed overnight at $4^{\circ} \mathrm{C}$ under constant agitation. Isolation of antibody-target-complexes by Dynabeads was performed at $4{ }^{\circ} \mathrm{C}$ for $2 \mathrm{~h}$ under rotation. Afterwards, DNA was reverse-crosslinked, cleaned up by spin columns (included in the ChIP kit) and amplified with the 'GenomePlex Single Cell Whole Genome Amplification Kit' (Sigma Aldrich). Amplified DNA was purified by PCI precipitation and analyzed by qPCR. In qPCR, oligonucleotides were used to amplify a PCRfragment around the SOX2 binding site (on target) and 
a PCR-fragment within the same gene, but without a SOX2 binding site (off target). Each qPCR analysis was performed in 3 technical replicates. For antibody and primer details see Tables S1 and S2.

\section{Quantification of DNA methylation levels}

$5 \mathrm{mC}$ levels were quantified as described previously [8]. For quantification, the 'MethylFlash Methylated DNA Quantification Kit (Colorimetric)’ (Epigentek, via BioCat, Heidelberg, Germany) was used. The experiment was performed according to the manual. For each measurement, $200 \mathrm{ng}$ of genomic DNA were analyzed. Each sample was analyzed in 8 technical replicates. DNA methylation levels were calculated according to the manual using the relative quantification method. For calculation and normalization a positive (methylated polynucleotide containing $50 \% 5 \mathrm{mC} ; n=3$ ) and negative (unmethylated polynucleotide containing $50 \%$ cytosine; $n=3$ ) control provided in the kit were included.

\section{Xenotransplantation of GCC cell lines}

Xenotransplantation was performed as described previously $[10,30] .1 \times 10^{7}$ cells in $500 \mu \mathrm{l}$ of $4^{\circ} \mathrm{C}$ cold Matrigel (BD, Heidelberg, Germany) were injected into the flank of CD1 nude mice. For 1 week of in vivo growth, 1 mouse each was xenografted with TCam-2- $\triangle \mathrm{SOX} 2$ or parental TCam-2 cells. For 6 weeks of in vivo growth, 5 mice were xenografted with TCam-2- $\Delta \mathrm{SOX} 2$ clones (1-5) and 1 mice each was xenografted with parental TCam-2 or 2102EP cells.

\section{Illumina HT-12v4 expression microarray}

The Illumina expression microarray analysis was performed as published [10]. Samples were processed on Illumina's (San Diego, CA, USA) human, HT-12v4' bead chips. All data were analyzed using, Bioconductor R' (www.bioconductor.org). A subset quantile normalization approach developed by N. Touleimat and J. Tost was applied [32]. Expression values were quantile normalized using the limma' software package ('Linear Models for Microarray Data', www.bioconductor.org). 6 tumor tissues from TCam-2- $\triangle$ SOX2 clones (1 after 1 week, 5 after 6 weeks), 2 tumor tissue from parental TCam-2 (1 after 1 week, 1 after 6 weeks), 1 from 2102EP grown for 6 weeks and 1 sample from in vitro cultivated TCam-2 was analyzed. Microarray data is publically available via GEO (ncbi.nlm.nih.gov/geo/) (GSE79065).

\section{Affymetrix expression microarray analysis of GCC tissues}

The whole procedure has already been published [21]. The array was reanalyzed in context of this study. In total, 4 seminoma and 7 non-seminoma tissues (teratoma, mixed non-seminoma) were analyzed. To identify genes differentially expressed between seminomas and nonseminomas, normalized, $\log _{2}$-transformed and averaged gene expression intensities of non-seminoma tissues were substracted from averaged seminoma tissues. All genes with a fold change $\geq \log _{2} 1.5$ were considered as significantly deregulated (Data S1 D).

\section{STRING analysis and Venn diagrams}

STRING protein-interaction-prediction were performed online using default settings (string-db. org) [33]. Venn diagrams were generated using 'Venny' (bioinfogp.cnb.csic.es/tools/venny).

\section{ACKNOWLEDGMENTS}

We kindly thank Blanca Randel, Gaby Beine, Susanne Steiner and Barabara Reddemann for technical assistance. Furthermore, we would like to thank Beatrice Weykopf (UKB, Life \& Brain, Bonn, Germany) for providing the FOXA2 antibody.

\section{CONFLICTS OF INTEREST}

The authors declare no conflicts of interest.

\section{FUNDING}

This study was supported by the DFG to HS (Scho503/17-1).

\section{REFERENCES}

1. Oosterhuis JW, Looijenga LHJ. Testicular germ-cell tumours in a broader perspective. Nat Rev Cancer. 2005; 5:210-22.

2. Berney DM, Looijenga L, Idrees M, Oosterhuis JW, Rajpert-De Meyts E, Ulbright TM, Skakkebaek NE. Germ cell neoplasia in situ (GCNIS): evolution of the current nomenclature for testicular pre-invasive germ cell malignancy. Histopathology. 2016; 69:7-10. doi: 10.1111/ his. 12958.

3. Kristensen DG, Skakkebk NE, Rajpert-de-Meyts E, Almstrup K. Epigenetic features of testicular germ cell tumours in relation to epigenetic characteristics of foetal germ cells. Int J Dev Biol. 2013;57:309-17.

4. de Jong J, Stoop H, Gillis A, van Gurp R, van de Geijn G-J, Boer M de, Hersmus R, Saunders P, Anderson RA, Oosterhuis JW, Looijenga L. Differential expression of SOX17 and SOX2 in germ cells and stem cells has biological and clinical implications. J Pathol. 2008; 215:21-30.

5. Gillis AJM, Stoop H, Biermann K, van Gurp RJHLM, Swartzman E, Cribbes S, Ferlinz A, Shannon M, Oosterhuis JW, Looijenga LHJ. Expression and interdependencies of pluripotency factors LIN28, OCT3/4, 
NANOG and SOX2 in human testicular germ cells and tumours of the testis. International Journal of Andrology. 2011; 34:e160-74.

6. Biermann K, Heukamp LC, Steger K, Zhou H, Franke FE, Sonnack V, Brehm R, Berg J, Bastian PJ, Muller SC, WangEckert L, Buettner R. Genome-wide expression profiling reveals new insights into pathogenesis and progression of testicular germ cell tumors. Cancer genomics \& proteomics. 2007; 4:359-67.

7. Wermann H, Stoop H, Gillis AJ, Honecker F, Van Gurp RJ, Ammerpohl O, Richter J, Oosterhuis JW, Bokemeyer C, Looijenga LH. Global DNA methylation in fetal human germ cells and germ cell tumours: association with differentiation and cisplatin resistance. J Pathol. 221:433-42.

8. Nettersheim D, Heukamp LC, Fronhoffs F, Grewe MJ, Haas N, Waha A, Honecker F, Waha A, Kristiansen G, Schorle H. Analysis of TET expression/activity and 5 $\mathrm{mC}$ oxidation during normal and malignant germ cell development. PLoS ONE. 2013; 8:e82881.

9. McGlynn KA, Devesa SS, Graubard BI, Castle PE. Increasing Incidence of Testicular Germ Cell Tumors Among Black Men in the United States. Journal of Clinical Oncology. 2005; 23:5757-61.

10. Nettersheim D, Jostes S, Sharma R, Schneider S, Hofmann A, Ferreira HJ, Hoffmann P, Kristiansen G, Esteller MB, Schorle H. BMP Inhibition in Seminomas Initiates Acquisition of Pluripotency via NODAL Signaling Resulting in Reprogramming to an Embryonal Carcinoma. PLoS Genet. 2015; $11: \mathrm{e} 1005415$.

11. Eckert D, Biermann K, Nettersheim D, Gillis AJ, Steger K, Jäck H-M, Müller AM, Looijenga LH, Schorle H. Expression of BLIMP1/PRMT5 and concurrent histone $\mathrm{H} 2 \mathrm{~A} / \mathrm{H} 4$ arginine 3 dimethylation in fetal germ cells, CIS/ IGCNU and germ cell tumors. BMC Dev Biol. 2008; 8:106-11.

12. Ohinata Y, Payer B, O'Carroll D, Ancelin K, Ono Y, Sano M, Barton SC, Obukhanych T, Nussenzweig M, Tarakhovsky A, Saitou M, Surani MA. Blimp1 is a critical determinant of the germ cell lineage in mice. Nature. 2005; 436:207-13.

13. Ancelin K, Lange UC, Hajkova P, Schneider R, Bannister AJ, Kouzarides T, Surani MA. Blimp1 associates with Prmt5 and directs histone arginine methylation in mouse germ cells. Nature Cell Biology. 2006; 8:623-30.

14. Cattoglio C, Zhang ET, Grubisic I, Chiba K, Fong YW, Tjian R. Functional and mechanistic studies of XPC DNArepair complex as transcriptional coactivator in embryonic stem cells. Proc Natl Acad Sci U S A. 2015; 112:E2317-26.

15. Rodda DJ. Transcriptional Regulation of Nanog by OCT4 and SOX2. J Biol Chem. 2005; 280:24731-7.

16. Pan G, Thomson JA. Nanog and transcriptional networks in embryonic stem cell pluripotency. Cell Research. 2007; 17:42-9.
17. Masui S, Nakatake Y, Toyooka Y, Shimosato D, Yagi R, Takahashi K, Okochi H, Okuda A, Matoba R, Sharov AA, Ko MSH, Niwa H. Pluripotency governed by Sox 2 via regulation of Oct $3 / 4$ expression in mouse embryonic stem cells. Nature Cell Biology. 2007; 9:625-35.

18. Sharov AA, Masui S, Sharova LV, Piao Y, Aiba K, Matoba R, Xin L, Niwa H, Ko MS. Identification of Pou5f1, Sox2, and Nanog downstream target genes with statistical confidence by applying a novel algorithm to time course microarray and genome-wide chromatin immunoprecipitation data. BMC Genomics. 2008; 9:269-19.

19. Eckert D, Nettersheim D, Heukamp LC, Kitazawa S, Biermann K, Schorle H. TCam-2 but not JKT-1 cells resemble seminoma in cell culture. Cell and Tissue Research. 2008; 331:529-38.

20. Nettersheim D, Gillis AJ, Looijenga LH, Schorle H. TGFbeta1, EGF and FGF4 synergistically induce differentiation of the seminoma cell line TCam-2 into a cell type resembling mixed non-seminoma. Int J Androl. 2011; 34:e189-203.

21. Eckert D, Nettersheim D, Heukamp LC, Kitazawa S, Biermann K, Schorle H. TCam-2 but not JKT-1 cells resemble seminoma in cell culture. Cell and Tissue Research. 2008; 331:529-38.

22. Sekiya T, Muthurajan UM, Luger K, Tulin AV, Zaret KS. Nucleosome-binding affinity as a primary determinant of the nuclear mobility of the pioneer transcription factor FoxA. Genes \& Development. 2009; 23:804-9.

23. Gosalia N, Yang R, Kerschner JL, Harris A. FOXA2 regulates a network of genes involved in critical functions of human intestinal epithelial cells. Physiol Genomics. 2015; 47:290-7.

24. Watts JA, Zhang C, Klein-Szanto AJ, Kormish JD, Fu J, Zhang MQ, Zaret KS. Study of FoxA pioneer factor at silent genes reveals Rfx-repressed enhancer at $\mathrm{Cdx} 2$ and a potential indicator of esophageal adenocarcinoma development. Reid B, editor. PLoS Genet. 2011; 7:e1002277.

25. Verschuur M, de Jong M, Felida L, de Maat MPM, Vos HL. A hepatocyte nuclear factor-3 site in the fibrinogen beta promoter is important for interleukin 6-induced expression, and its activity is influenced by the adjacent $-148 \mathrm{C} / \mathrm{T}$ polymorphism. J Biol Chem. 2005; 280:16763-71.

26. Kaestner KH. The FoxA factors in organogenesis and differentiation. Current Opinion in Genetics \& Development. 2010; 20:527-32.

27. Kerschner JL, Gosalia N, Leir S-H, Harris A. Chromatin remodeling mediated by the FOXA1/A2 transcription factors activates CFTR expression in intestinal epithelial cells. Epigenetics. 2014; 9:557-65.

28. Friedman JR, Kaestner KH. The Foxa family of transcription factors in development and metabolism. Cellular and molecular life sciences. 2006; 63:2317-28. 
29. Sugathan A, Waxman DJ. Genome-wide analysis of chromatin states reveals distinct mechanisms of sexdependent gene regulation in male and female mouse liver. Molecular and Cellular Biology. 2013; 33:3594-610.

30. Nettersheim D, Westernströer B, Haas N, Leinhaas A, Brüstle O, Schlatt S, Schorle H. Establishment of a versatile seminoma model indicates cellular plasticity of germ cell tumor cells. Genes Chromosom Cancer. 2012; 51:717-26.

31. Tu SM, Bilen MA, Hess KR, Broaddus RR, Kopetz S, Wei C, Pagliaro LC, Karama JA, Ward JF, Wood CG, Rao P, Tu ZH, General R, Chen AH, Nieto YL, Yeung S-CJ, Lin SH, Logothetis CJ, Pisters LL. Intratumoral heterogeneity: Role of differentiation in a potentially lethal phenotype of testicular cancer. Cancer. 2016; 122:1836-43.
32. Touleimat $\mathrm{N}$, Tost $\mathrm{J}$. Complete pipeline for Infinium (RHuman Methylation 450K BeadChip data processing using subset quantile normalization for accurate DNA methylation estimation. Epigenomics. 2012; 4:325-41.

33. Szklarczyk D, Franceschini A, Wyder S, Forslund K, Heller D, Huerta-Cepas J, Simonovic M, Roth A, Santos A, Tsafou KP, Kuhn M, Bork P, Jensen LJ, Mering von C. STRING v10: protein-protein interaction networks, integrated over the tree of life. Nucleic Acids Research. 2015; 43:D447-52. 